\title{
Cómo comunicar la investigación desde la academia
}

How to communicate research from the academy

DOI 10.5377/rct.v0i24.7872

Claudia Nieto Anderson ${ }^{1}$

Laura Andrea Aceituno ${ }^{2}$

En el mundo de la ciencia existen algunos términos fascinantes que las autoras, como profesionales del área de la comunicación y el periodismo, procuran impulsar desde su quehacer diario.

El principal término al que haremos referencia es comunicación científica, con la aclaración de que cada uno de los conceptos derivados está relacionado con la comunicación de la ciencia y cómo los hallazgos de esta última pueden ser transmitidos efectivamente a todos los públicos que sea necesario. Este artículo se propone como una exploración de estos conceptos que podría ser útil para iniciar conversaciones, debates y reflexiones en los espacios universitarios en que nos movemos.

\section{¿Qué es la comunicación científica?}

La comunicación es esencial a la naturaleza y a la práctica de la ciencia, por tanto, es el núcleo de la ciencia moderna (Gregory, 2015). Con esta breve definición -que no hace referencia a su etimología- se pretende puntualizar y entender dos términos importantes para la ciencia: la divulgación y la publicación científica.

Aunque la definición sobre ciencia está caracterizada en la actualidad, por el seguimiento de un método, la construcción de hipótesis, la experimentación para exponer resultados y la comprobación de teorías (Fara, 2015); antes, la práctica de la ciencia no contaba con estos «parámetros universales». No fue sino hasta el comienzo de las revoluciones, como la científica y la industrial, que inició la evolución de las prácticas y procesos de difusión de la ciencia.

Los procesos de difusión de la ciencia fueron con el fin de que los resultados de los experimentos científicos, realizados hace varios siglos, pudieran ser entendidos

\footnotetext{
${ }^{1}$ Trabaja en la Dirección de Investigación Científica Universitaria en la Universidad Nacional Autónoma de Honduras. Es Licenciada en Comunicación y Publicidad, con Maestría en Enseñanza de Lenguas y cursa la Maestría en Comunicación Corporativa.

${ }^{2}$ Es Licenciada en Periodismo, con Maestría en Historia de la Ciencia y Comunicación Científica
} 
colectivamente por un público. A su vez, era preciso contar con una audiencia, un reporte y una examinación de terceros (Gregory, 2015). En la historia del Occidente, la comunicación de la ciencia o la divulgación científica tiene su inicio desde antes de la propia ciencia institucionalizada en su sentido moderno. Los propios estudiosos 0 experimentadores eran los encargados en la divulgación de sus conocimientos y metodologías empleadas, a modo de difundir sus investigaciones; y del mismo modo, hacer entender al público sus hallazgos. Como, por ejemplo, los eruditos en filosofía natural o los primeros astrónomos como lo fueron los griegos y árabes; los cirujanos-barberos en la medicina medieval, entre otros.

\section{¿Cuál es la diferencia conceptual entre la divulgación y publicación de la ciencia?}

La conceptualización de estos términos viene, en parte, acompañada por las prácticas de investigación y sus formatos de difusión. Históricamente se señala que fue en el siglo XVII cuando los escritos o publicaciones comienzan a institucionalizarse con las denominadas «Academias de las Ciencias», por ejemplo, Academia dei Lincei en 1603 y la Royal Society en 1662.

La divulgación comienza a surgir a través de los museos, jardines botánicos y observatorios astronómicos. Estas instituciones y estrategias de divulgación permitían la publicidad de los primeros científicos. De igual manera, la puesta común de un vocabulario, metodologías y resultados aparecen con las primeras revistas científicas contemporáneas: Nature en 1869 y Science en 1880 (Sanz Merino, 2011).

La comunicación social de la ciencia o la divulgación repercutió entre los siglos XIX y $X X$, este último considerado como la «época dorada de la divulgación científica». Ya no solamente se presentaba el interés del público, sino la transformación cultural que experimentó la sociedad con nuevos formatos para la difusión de la ciencia.

En ese sentido, la divulgación científica no consiste en «hacer comprender al lego de la red conceptual científica», sino en encontrar y utilizar los términos y los recursos idóneos que puedan dotar de sentido los conceptos concretos que se deben transmitir (Moreno Castro \& Gil Pérez, 2014). La divulgación, viene a corresponder a aquellos que han tenido una formación especializada en ciertas ramas de la ciencia.

Las publicaciones científicas, por otro lado, son el trabajo realizado por los especialistas -investigadores o científicos- que siguen un marco teórico-metodológico, postulación de hipótesis, experimentación, comprobación de teorías y exposición de resultados. A diferencia de la divulgación, estos siguen un proceso de revisión por pares 
previo a sus publicaciones en revistas especializadas.

Corresponde a las publicaciones la generación de los conceptos universales. En otros términos, un artículo o publicación científica se origina cuando es comunicable. Es decir, cuando es posible su apertura en la publicación cuando son verificables los datos empíricos y las hipótesis científicas (Miyahira, 2018).

La publicación científica se define, en resumidas palabras, como el escrito que describe los resultados de una investigación. Esta investigación previamente debe ser redactada -y publicada- de acuerdo con la práctica editorial, siguiendo los parámetros de la ética científica.

\section{¿Qué métodos de divulgación científica pueden ser utilizados?}

La divulgación pretende ayudar al público a entender la ciencia, a tomar decisiones informadas como ciudadanos y en la generación de políticas responsables.

Como la Royal Society recomendó desde 1985 para el Reino Unido, la ciencia y la tecnología permea nuestras vidas diarias, por lo que la comprensión de estas es importante para la satisfacción personal y bienestar de los individuos, para la participación de los ciudadanos en una sociedad democrática, para los trabajadores cuyas habilidades y ocupaciones tienen algún involucramiento científico o técnico, para las personas empleadas cuyas decisiones tienen efecto en el medio ambiente (1985). Del mismo modo, busca que los tomadores de decisiones mejoren la orientación de las políticas públicas.

Los medios de comunicación son los principales espacios en la participación de la divulgación científica. El periodismo científico es el área especializada que utiliza un lenguaje más claro, sin la utilización de tecnicismos, generando un relato que permite dar una información general dirigida a todos los públicos no especializados. En el informe citado de la Royal Society (1985) a los medios de comunicación masiva se les recomendaba que sus periodistas comprendieran el abordaje que los científicos hacen en sus campos, asimismo solicitaba a los editores y miembros con avanzada experiencia en los equipos de redacción que produjeran más y mejor material acerca de ciencia y tecnología, entre otras recomendaciones.

En un estudio, cuya muestra fueron periódicos de nueve países de América Latina, (Almeida, Ramalho, Buys, \& Massarini, 2011), se presenta una breve observación sobre el impacto de la divulgación de la ciencia en la sociedad. En primer lugar, la 
pauta generada por la divulgación de la ciencia creada en países desarrollados y no por la ciencia creada por países vecinos.

Y, en segundo lugar, por la dificultad de acceso causada por las reducidas estructuras de asesoría de prensa en las universidades y centros de investigación. Estas dos estructuras -no señaladas como mediadoras- vienen a ser una especie de consejeras, tanto para generar una cultura de hechos, como una fuente de información fiable y así, poder obtener resultados respecto a la divulgación.

Otros medios corresponden a la museología, los documentales, el cine, los podcasts, monólogos, ilustración científica y otros espacios de difusión orientados desde la academia, institutos de investigación y museos de ciencia a la sociedad.

\section{¿Qué formas de publicación científica existen?}

Las publicaciones científicas presentan un formato más elaborado y estricto para su conocimiento, incluso su difusión presenta límites. En primer lugar, estas son dirigidas a un público más especializado, por lo tanto, la difusión les corresponde a revistas especializadas donde requiere evaluación de pares externos. Es decir, investigadores o científicos de otras editoriales, universidades o institutos de investigación.

El acceso a estas revistas especializadas presenta una dificultad para algunos grupos de personas que se dedican a la investigación. En primera instancia, porque estas tienen un costo. Lo mismo sucede al momento de realizar una publicación, nos referimos a que, entre más renombre tiene una revista, el proceso de publicación es más complejo y la investigación debe tener una importancia considerable a nivel internacional.

\section{¿Para qué utilizar correctamente los términos?}

En los apartados anteriores se ha explicado las diferencias de términos. Si bien el uso de cada uno de ellos no puede ser controlado en todos los espacios, debemos enfatizar en la importancia de denominar correctamente cada uno de estos por aspectos como la planificación, la asignación de recursos, la investigación en comunicación científica 0 el seguimiento y evaluación de las estrategias y actividades que esta conlleva.

La exposición realizada hasta este punto es necesaria para aspectos normativos, estratégicos y técnicos. Hay que tener en cuenta que, si bien se debe animar a una mayor clarificación y delimitación conceptual, los usuarios más frecuentes de los 
términos no tomarán decisiones sobre cómo se gestiona la comunicación científica y esto significa que luchar constantemente por el buen uso de los términos es menos importante que informar adecuadamente a todos los públicos posibles con los resultados de la investigación científica.

Los aspectos prácticos de comunicar la ciencia adquieren relevancia en una época marcada por la viralidad de noticias e información que no está basada en evidencia. Es decir, los términos no son el objetivo de la discusión sino el medio para que la ciencia sea accesible más allá del núcleo que conforman los científicos. De ahí que reconocemos que no tiene sentido hacer ciencia y no contarle a la sociedad los hallazgos y avances.

Este enlace entre el uso de los términos en los espacios de toma de decisión y de planificación de la investigación es lo que nos lleva a preocuparnos porque se divulguen adecuadamente los avances de la ciencia y de la tecnología. Al menos en Honduras podemos hacer suposiciones generales sobre la actitud que tiene la población acerca de la ciencia, pero aparte de unas bien intencionadas opiniones es posible que no encontremos datos o información sobre la alfabetización científica y tecnológica de los ciudadanos. Esta información permitiría tomar decisiones en todos los niveles educativos, así como en el país en general para apuntar a un conocimiento mínimo generalizado que compartamos los hondureños.

Si se tiene en cuenta que son los políticos quienes deciden sobre el gasto público en I+D+i

${ }^{3}$ y que se encuentra vinculado directamente con la economía nacional y regional, la información sobre ello debería tener mayor relevancia en las sociedades contemporáneas. Los líderes de opinión y el público en general debieran aprender más y mejor el sentido de la I+D+i, conocer con mayor rapidez sus resultados y tomar conciencia de que las inversiones en este campo son útiles para todos. (Calvo Hernando \& Calvo Roy, 2011)

\section{Evaluación de la comunicación científica}

Una vez asumidas la planificación y la ejecución de políticas y actividades de comunicación científica, también se debe evaluar su impacto. Este, en términos de la sociedad, debería implicar que haya unos conocimientos mínimos compartidos entre todos los ciudadanos para la vida en sociedad. La alfabetización científica cívica ha sido un tema de amplio estudio y debate desde hace varias décadas en otras latitudes:

\footnotetext{
${ }^{3}$ I+D+i se utiliza para abreviar los términos investigación científica, desarrollo tecnológico e innovación.
} 
La alfabetización científica cívica [...] se definió como el nivel de conocimiento de principios básicos de la ciencia y la tecnología suficiente como para leer información sobre el tema en un periódico o en una revista, y para entender los argumentos que intervienen en una controversia. Un nivel que permitiría a un individuo desenvolverse como ciudadano en la sociedad moderna, en la que la ciencia es permeable en todos los ámbitos, o ejercer sus responsabilidades cívicas, tal y como podían desear las agrupaciones de ciudadanos y la comunidad científica.

[...] El umbral mínimo para alcanzar la alfabetización científica cívica aumenta progresivamente con el avance de la ciencia, y su determinación dependerá de lo que los expertos consideren adecuado. (Montañés Perales, 2011)

Existen diversos abordajes para hacer comunicación científica. En 2010, el gobierno del Reino Unido publicó una herramienta conversacional para motivar la comprensión de la ciencia y la tecnología. Esta herramienta se presenta como un triángulo en el que se encuentran transmitir, recibir y colaborar, y bajo este modelo se asume que cualquier actividad de comunicación de la ciencia debe combinar estas tres actividades según las necesidades de la audiencia y del científico involucrados (Jucan \& Jucan, 2014).

Por ejemplo, se puede determinar qué tan basados en evidencia fueron diseñados los afiches de campañas de salud que son colgados en hospitales, evaluar el rigor periodístico en las noticias basadas en investigaciones, actualizar -y corregir, si es necesario- los textos escolares según avanza la ciencia, y la evaluación deberá tener siempre un componente cualitativo amplio que brinde información sobre cuánto conoce la sociedad sobre la ciencia y la tecnología.

En conclusión, corresponde a la academia, las estructuras de investigación nacionales, los comunicadores sociales y los medios de comunicación el fortalecer la comunicación de la ciencia a través del trabajo colectivo, realizando consensos para llevar a cabo iniciativas para la construcción de una cultura que procure aceptar información basada en hechos y evidencia. 


\section{REFERENCIAS BIBLIOGRÁFICAS}

Almeida, C., Ramalho, M., Buys, B., \& Massarini, L. (2011). La cobertura de la ciencia en América Latina: estudio de periódicos de élite en nueve países de la región. En C. Moreno Castro (Ed.), Periodismo y divulgación científica. Tendencias en el ámbito iberoamericano. (p. 379). Madrid, España: OEI, AECID, Grupo Editorial Siglo XXI.

Calvo Hernando, M., \& Calvo Roy, A. (2011). De la divulgación científica a la ciencia mediática. En C. Moreno Castro (Ed.), Periodismo y divulgación científica. Tendencias en el ámbito iberoamericano. (p. 379). Madrid, España: OEI, AECID, Grupo Editorial Siglo $\mathrm{XXI}$.

Fara, P. (2015). ¿Qué es la ciencia? Confusiones de una historiadora. Mètode Science Studies Journal, (5), 45-49. https://doi.org/10.7203/metode.84.3915

Gregory, J. (2015). Science Communication. En J. D. Wright, International Encyclopedia of the Social \& Behavioral Sciences (Segunda edición, Vol. 21, pp. 219-224). Recuperado de http://dx.doi.org/10.1016/B978-0-08-097086-8.95088-8

Jucan, M. S., \& Jucan, C. N. (2014). The power of science communication. Procedia - Social and Behavioral Sciences, 149, 461-466. https://doi.org/10.1016/j.sbspro.2014.08.288

Miyahira, J. (2018). Publicación científica. Revista Médica Herediana, 9(1), 1-2. Recuperado de www.upch.edu.pe/vrinve/dugic/revistas/index.php/RMH/article/view/561/528

Montañés Perales, Ó. (2011). Percepción social de la ciencia y la tecnología. En C. Moreno Castro (Ed.), Periodismo y divulgación científica. Tendencias en el ámbito iberoamericano. (p. 379). Madrid, España: OEI, AECID, Grupo Editorial Siglo XXI.

Moreno Castro, C., \& Gil Pérez, A. (2014). ¿Periodismo diletante o ciencia mediática? La metamorfosis del artículo científico en noticia de prensa. En C. Barrio Alonso \& S. Cáceres Gómez (Eds.), Fronteras de la ciencia: dilemas (p. 180). Madrid: Biblioteca Nueva.

Sanz Merino, N. (2011). La perspectiva CTS en el estudio y reflexión sobre la Comunicación Social de la Ciencia y la Tecnología. En C. Moreno Castro (Ed.), Periodismo y divulgación científica. Tendencias en el ámbito iberoamericano. (p. 379). Madrid, España: OEI, AECID, Grupo Editorial Siglo XXI.

The Royal Society. (1985). The Public Understanding of Science (p. 46). Recuperado de https://royalsociety.org/-/media/Royal_Society_Content/policy/publications/1985/10700.pdf 